\title{
Uniform Heating Model Analysis for the Formation Process of Aluminium Foam by Spot-Type Halogen Lamp
}

\author{
R. Nagahiro ${ }^{1,2^{*}}$, N. Rosli ${ }^{2}$, Y. Hangai ${ }^{1}$, A. Yano $^{1}$ and K. Amagai $^{1}$ \\ ${ }^{1}$ Graduate School of Science and Technology, Gunma University, \\ Kiryu 376-8515, Japan \\ *Email: t14302079@gunma-u.ac.jp \\ Phone: +81277301513; Fax: +81277301508 \\ ${ }^{2}$ Faculty of Manufacturing Engineering, Universiti Malaysia Pahang, \\ 26600, Pekan, Pahang, Malaysia
}

\begin{abstract}
Heat treatment by heating furnace has limited the capability of heat treatment processes to control the desired heating volume and products' sizes. This study introduced a heat treatment process using light heat sourced from a spot-type halogen lamp, with the aim of clarifying the thermal phenomenon of aluminium (Al) foam. Here, the temperature and deformation of $\mathrm{Al}$ foam were observed experimentally. In addition, thermal analysis was conducted numerically using a uniform heating model by neglecting the energy consumption of the blowing agent and deformation and thermal conductivity of Al. The experimental results revealed that the precursor initially formed at almost the same time as the phase change. The numerical results almost corresponded with the experimental results until the melting point of $\mathrm{Al}$, after which a slight disagreement was observed. Moreover, the phase transformation appeared slightly earlier as a result of neglecting the deformation of $\mathrm{Al}$ foam and energy consumption of the blowing agent. Although the formation of $\mathrm{Al}$ foam cannot be expressed perfectly using the proposed uniform heating model, a qualitative phenomenon of such formation was successfully explained. In the future, the effects of deformation and energy consumption of the blowing agent and the thermal conductivity of $\mathrm{Al}$ can be considered in the proposed uniform heating model.
\end{abstract}

Keywords: Aluminium foam; halogen lamp; light heating; numerical thermal analysis.

\section{NOMENCLATURE}

$Q \quad$ input heat per second per unit area $\left[\mathrm{J} / \mathrm{s} \cdot \mathrm{m}^{2}\right]$

$A \quad$ top surface area of precursor $\left[\mathrm{m}^{2}\right]$

$T \quad$ temperature of precursor $[\mathrm{K}]$

$T_{0} \quad$ temperature of surrounding wall [K]

$\varepsilon \quad$ absorption or emission coefficient [-]

$\sigma \quad$ Stefan-Boltzmann's constant $\left[\mathrm{W} / \mathrm{m}^{2} \cdot \mathrm{K}^{4}\right]$

$A^{\prime} \quad$ one lateral surface of precursor $\left[\mathrm{m}^{2}\right]$

$\alpha_{m, h}$ mean heat transfer coefficient of horizontal plane $\left[\mathrm{W} / \mathrm{m}^{2} \cdot \mathrm{K}^{4}\right]$

$T_{w} \quad$ temperature of precursor surface $[\mathrm{K}]$

$T_{\infty} \quad$ atmospheric temperature $[\mathrm{K}]$

$\alpha_{m, v} \quad$ mean heat transfer coefficient of vertical plane $\left[\mathrm{W} / \mathrm{m}^{2} \cdot \mathrm{K}^{4}\right]$

$L_{a} \quad$ latent heat $[\mathrm{J} / \mathrm{kg}]$ 


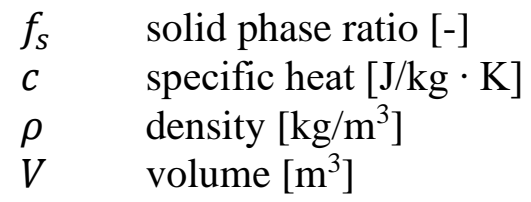

\section{INTRODUCTION}

Aluminium ( $\mathrm{Al}$ ) foam is well known for its excellent properties such as its lightweight by the low density, noise reduction, and high shock absorption rate, making it an increasingly interesting topic for exploration [1 - 3]. For more than 60 decades, various application attempts on $\mathrm{Al}$ foam in the industrial field were made, especially in the automotive sector. Nonetheless, technical elements ascertaining the cost, size limitation, and so on prevented the smooth applicability of $\mathrm{Al}$ foam in the automotive sector. One of the most important processes in the fabrication of $\mathrm{Al}$ foam is heat treatment [1 - 5], typically conducted with the use of a heating furnace that consumes a large amount of the heat source to be able to heat the furnace spaces completely. Additionally, time consumption becomes considerably longer due to the mandatory procedure of preheating the furnace; thus, controlling the desired heating volume exerted to the material being heated becomes difficult. Moreover, since the size of the material being formed mainly depends on the capacity of the furnace, the manufacturing capability is limited in size and quantity. This study introduces a new heat treatment process using light heat sourced from a halogen lamp. The advantage of this method lies in the reduction of energy consumption where a smaller heat volume is required in a shorter time and at a lower cost. This method also permits the observation of the fabrication behaviour of $\mathrm{Al}$ foam by using a video camera during the heat treatment process, which is commonly unattainable under the usage of a heating furnace due to the existence of its surrounding wall.

Furthermore, clarifying the occurrence of a thermal phenomenon during the heat treatment process is challenging as the process involves complicated events such as heat transfer, deformation, and a chemical reaction $[6,7]$. Although there has been an ample number of $\mathrm{Al}$ foam analysis conducted in the recent years [8, 9], most of these were merely adapted to the final product of $\mathrm{Al}$ foam because making a detailed description of the thermal analysis during the formation process is difficult. Nonetheless, as such a phenomenon should be essentially clarified, this study provides an attempt to elucidate the thermal analysis of $\mathrm{Al}$ foam. Of the existing $\mathrm{Al}$ foam fabrication methods in the field of metal foam, the precursor method is employed in this study [10]. Normally, $\mathrm{Al}$ foam can be deformed by a blowing agent that should be mixed into the base material prior to the conduct of heat treatment process. The blowing agent in precursor released hydrogen gas as soon as Al melts [11], which depends on the temperature and holding time [12]. Hydrogen gas produced from the Al foam surface during the heating process caused the $\mathrm{Al}$ foam to shrink after certain time and temperature. Moreover, the hydrogen gas that released from the thermal decomposition shrink together with the Al metal during the cooling phase, after the heating process. Thus, optimal time and heat condition are highly required to prevent such shrinking of $\mathrm{Al}$ foam. Up to the present, only a primitive value has been used in previous studies $[13,14]$ such as input heat value, distance between the lamp and material, and heating time.

Herein, the thermal phenomenon during the light heating process in $\mathrm{Al}$ foam is clarified in order to predict the optimal time and heat condition. A spot-type halogen lamp is used as the light heat source. During the experiment, the temperature and deformation of $\mathrm{Al}$ foam are observed by means of a thermocouple and a video camera, respectively. 
The thermal phenomenon is then clarified in detail based on the thermal analysis with a uniform heating model, which is a model that neglects the energy consumption of the blowing agent as well as the deformation and thermal conductivity of the material. The thermal analysis of this model is numerically simulated so as to clarify the thermal phenomenon of $\mathrm{Al}$ foam during the light heating process. This way, the optimal time and heat condition in various situations can also be predicted.

\section{METHODOLOGY}

\section{Experimental Setup}

Figure 1 shows a schematic illustration of the experimental setup for the heat treatment process in this study. The setup mainly consisted of a heating section, a controller part, and a measurement system. The heating section included a halogen lamp as the heat source, an elliptical reflector to concentrate light onto the test piece, and a cooling system to prevent the increase in temperature of the setup equipment in the heating section. The halogen lamp exhibited a maximum power supply of $2 \mathrm{~kW}$ with a single phase of $100 \mathrm{~V}$ and current of $20 \mathrm{~A}$. During the experiment, the voltage and current were set at $54 \mathrm{~V}$ and 9.0 A, respectively. The elliptical reflector was specially coated with gold to intensively gather the light source onto the test piece, to increase the reflection ratio, and maintain high energy efficiency. The focus range of the elliptical reflector was 45 to $50 \mathrm{~mm}$. Thus, the top surface of the test piece was set at $50 \mathrm{~mm}$ from the bottom of the elliptical reflector. The cooling system was supplied with water at a rate of approximately $1 \mathrm{~L} / \mathrm{min}$. For the controller part, a voltage transformer was employed to adjust the voltage, while a current meter was used to read the current value. For the measurement system, a K-type thermocouple was used to measure the temperature of the test piece. Simultaneously, deformation of the $\mathrm{Al}$ foam was recorded with a video camera. The recorded movie was then analysed through image analysis using ImageJ software.

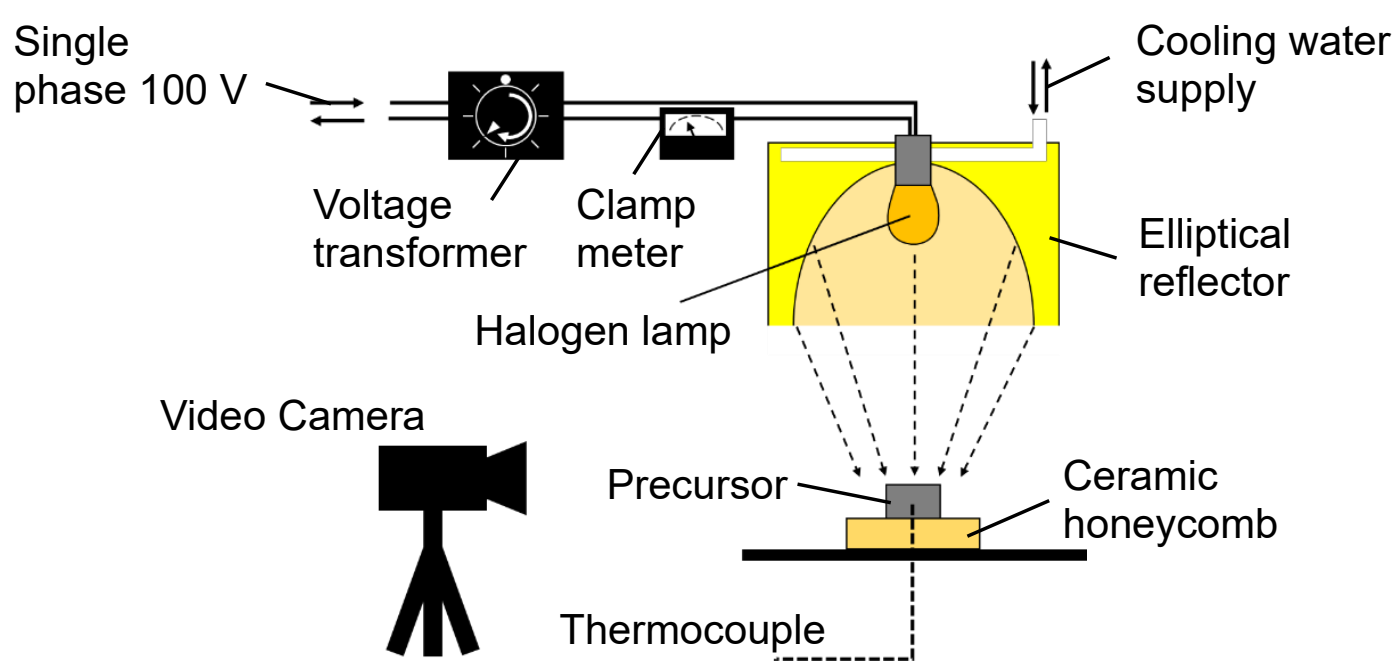

Figure 1. Schematic illustration of the experimental setup

An Al-Si-Cu alloy ADC12, as defined in Japanese Industrial Standard (JIS), diecasting plate was used as the base material. The chemical composition of the ADC12 used 
is presented in Table 1 . The solidus and liquidus temperatures of the alloy were $515^{\circ} \mathrm{C}$ and $580^{\circ} \mathrm{C}$, respectively. In this study, the friction stir welding (FSW) of the precursor method was employed to fabricate the $\mathrm{Al}$ foam [15].

Table 1. Chemical composition of ADC12 used in the present study.

\begin{tabular}{lccccccccc}
\hline Element & $\mathrm{Si}$ & $\mathrm{Fe}$ & $\mathrm{Cu}$ & $\mathrm{Mn}$ & $\mathrm{Mg}$ & $\mathrm{Zn}$ & $\mathrm{Ni}$ & $\mathrm{Sn}$ & $\mathrm{Al}$ \\
\hline mass $\%$ & 10.14 & 0.82 & 1.72 & 0.27 & 0.18 & 0.56 & 0.04 & 0.02 & $\mathrm{Bal}$. \\
\hline
\end{tabular}

Figure 2 shows the schematic illustration of the precursor fabrication and the principle of foam formation for this study. $\mathrm{TiH}_{2}(<45 \mu \mathrm{m}, 1$ mass $\%)<45 \mu \mathrm{m}, 1$ mass $\%$ and $\mathrm{Al}_{2} \mathrm{O}_{3}(\sim 1 \mu \mathrm{m}, 5$ mass $\% \sim 1 \mu \mathrm{m}, 5$ mass $\%)$ were used as the blowing and stabilisation agents, respectively. On one hand, $\mathrm{TiH}_{2}$ released hydrogen gas by thermal decomposition at $480{ }^{\circ} \mathrm{C}$ for $180 \mathrm{~min}$ [11]. Conversely, $\mathrm{Al}_{2} \mathrm{O}_{3}$ increased the $\mathrm{Al}$ viscosity in order to keep the thickness of the Al foam's cavity wall [16]. The test sample was prepared using the mulch pass process where the blowing and stabilisation agents were initially sandwiched by two Al plates before they were mixed together along with multiple lines using a high-speed rotating tool. Here, both the blowing and stabilisation agents can be considered to be homogeneously distributed in the material [17]. Subsequently, the sample was cut into small pieces of precursor in $15 \mathrm{~mm} \times 15 \mathrm{~mm} \times 6$ $\mathrm{mm} .15 \mathrm{~mm} \times 15 \mathrm{~mm} \times 6 \mathrm{~mm}$ At the centre of the bottom surface of the precursor, a hole was drilled in order to insert the thermocouple for temperature measurement. The hole dimension diameter and depth were 1.01 .0 and $3.0 \mathrm{~m} 3.0 \mathrm{~m}$, respectively.

(a)

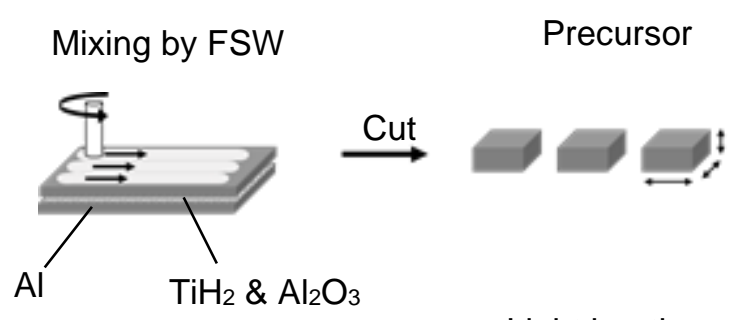

(b)

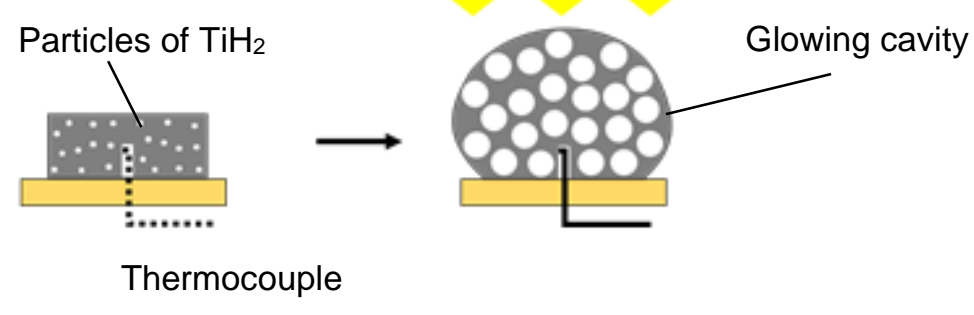

Figure 2. Schematic illustration of (a) precursor fabrication and (b) principle of foam formation.

\section{Theoretical Principle}

The spot-type halogen lamp used in this study was aimed at rapidly and uniformly increasing the temperature of the precursor as the heated object. However, this heating 
process involves complicated events such as heat transfer, deformation, and chemical reaction [18]. Therefore, the present study elucidates on the thermal phenomenon in the subsequent texts.

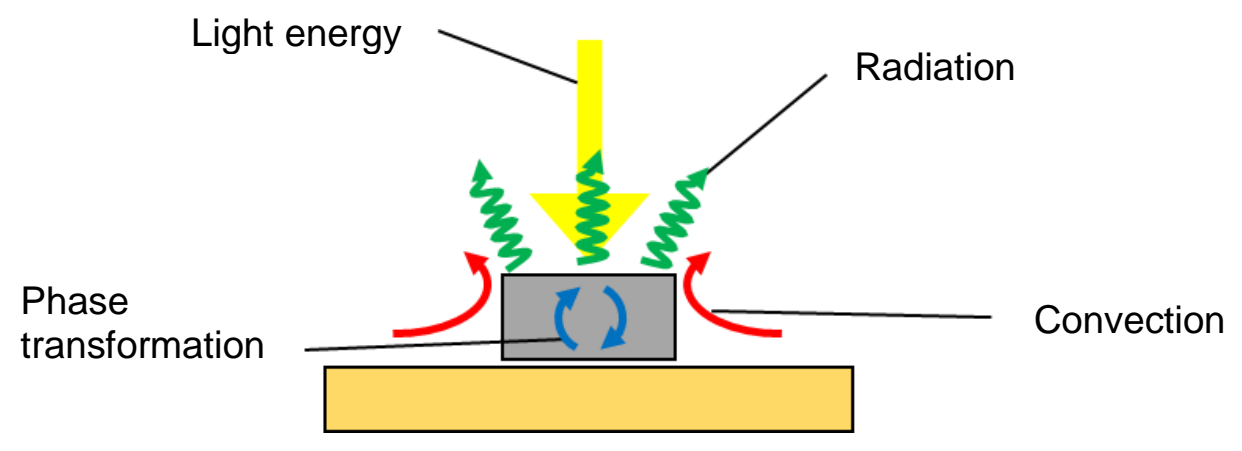

Figure 3. Illustration of the uniform heating model.

Here, it was assumed that the thermal conductivity of $\mathrm{Al}$ is extremely high and that of the heated object is extremely thin. As such, the internal thermal distribution of the heated object can be neglected, and hence, the temperature of the whole object is instantly constant. Moreover, the energy consumption of the blowing agent and deformation were neglected in this study as well. Even with the observable growing cavity, formulating the blowing agent is difficult, as the resulting equation is a function of pressure and verbosity of expansion. As an option, this matter is considered in a future experiment using an $\mathrm{x}$-ray system. The heated object was then placed on a ceramic plate, as shown in the Figure 1 and 2, to eliminate the effects of thermal loss. Thus, transporting heat energy in the uniform heating model mainly consisted of radiation by light or high temperature, convection, and phase transfer, as illustrated in Figure 3.

For the radiation term, the temperature of the heated object was raised by exposing the light of the halogen lamp to the top surface of the object. The emission coefficient, which is an important value in the radiation, was generally equal to the absorption coefficient. This absorption coefficient represents the absorption ratio of the light energy of the heated object. When heat loss from the surface of the heated object is much smaller than the area of the surrounding wall, the emitted heat amount is given by StefanBoltzmann's law. Because the heat amount was proportional to the emission or absorption coefficient and the fourth power of differential temperature in this law, the radiation term can be expressed by:

$$
\varepsilon A Q-A \sigma \varepsilon\left(T^{4}-T_{0}^{4}\right)-4 A^{\prime} \sigma \varepsilon\left(T^{4}-T_{0}^{4}\right)
$$

Due to the presence of temperature difference in this study, there is a need to consider convection occurrence. Here, the heat amount of convection that is expressed by the heat amount per unit area is determined by Newton's law of cooling. The density of the air around the heated object is changed depending on the heating or cooling conditions in the atmosphere, during which the fluid motion normally caused by gravity is called natural convection. The deformation is neglected by assuming that the shape of the heated object is consistent. Thus, the phenomenon can be classified into two types, namely, horizontal and vertical heat transfer planes. The horizontal heat transfer plane represents the heat transfer phenomenon at the top surface of the heated object, whereas the vertical heat transfer plane represents four lateral surfaces of the heated object. Consequently, the convection term can be expressed by: 


$$
-A \alpha_{m, h}\left(T_{w}-T_{\infty}\right)-4 A^{\prime} \alpha_{m, v}\left(T_{w}-T_{\infty}\right) .
$$

Finally, the thermal energy of the heated object is absorbed or emitted when the phase of the material changes from a solid into liquid condition, also called latent heat. As the object is heated by the halogen lamp, the effects of heat absorption due to the latent heat can be considered when the temperature is above the melting point. In this study, the latent heat must be converted into an energy changing rate since it is normally a material's property value. In order to express the energy changing rate, the solid phase ratio is employed. This solid-phase ratio is a proportion of the solid phase and the liquid phase, as determined by the condition figure by the principle of lever [19]. Specifically, latent heat multiplied by the solid phase ratio indicates the energy changing rate of the latent heat. Therefore, the equation of latent term is expressed by

$$
-\mathrm{L}_{\mathrm{a}} \mathrm{m}\left(\mathrm{df}_{\mathrm{s}} / \mathrm{dT}\right)(\mathrm{dT} / \mathrm{dt})
$$

This term only appears when the temperature of a material is higher than the Al melting point. The condition that expresses the relation of energy conservation during the phase transfer is described by:

$$
L_{a} m-\int_{t 1}^{t 2} L_{a} m\left(d f_{s} / d T\right) d t>0
$$

From these terms and energy conservation law, the equation of uniform heating model that indicates the time changing rate of temperature is described by:

$$
\begin{aligned}
\rho c V(d T / d t)= & \varepsilon A Q-A \sigma \varepsilon\left(T^{4}-T_{0}{ }^{4}\right)-4 A^{\prime} \sigma \varepsilon\left(T^{4}-T_{0}{ }^{4}\right) \\
& -A \alpha_{m, h}\left(T_{w}-T_{\infty}\right)-4 A^{\prime} \alpha_{m, v}\left(T_{w}-T_{\infty}\right)-L_{a} m\left(d f_{s} / d T\right)(d T / d t) .
\end{aligned}
$$

\section{RESULTS AND DISCUSSION}

\section{Experimental Results}

Heat treatment was applied to the precursor at the current of $9.0 \mathrm{~A}$, during which deformation of the $\mathrm{Al}$ foam was recorded by means of a video camera. The recorded video movie was then converted into images of every $5 \mathrm{~s}$ interval. Figure 4 shows the sample images of foam formation of the precursor at the (a) starting time, (b) starting time of foam formation, i.e., $50 \mathrm{~s}$, (c) in the middle of deformation, i.e., $90 \mathrm{~s}$, and (d) finishing time of foam formation.

From the selected images in the $5 \mathrm{~s}$ interval, the projected area of the precursor was determined using ImageJ software. The results of the projected area were compared with the temperature of the precursor measured by the thermocouple, as shown in Figure 5 . 

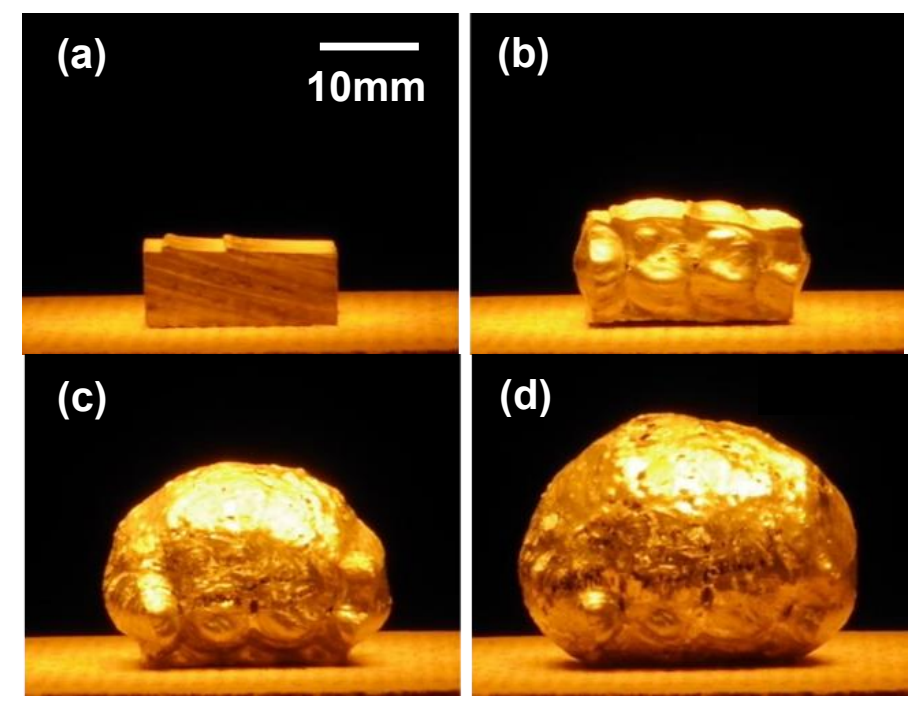

Figure 4. Foam formation of precursor at (a) 0, (b) 50, (c) 90 and (d) $120 \mathrm{~s}$ during light heating.

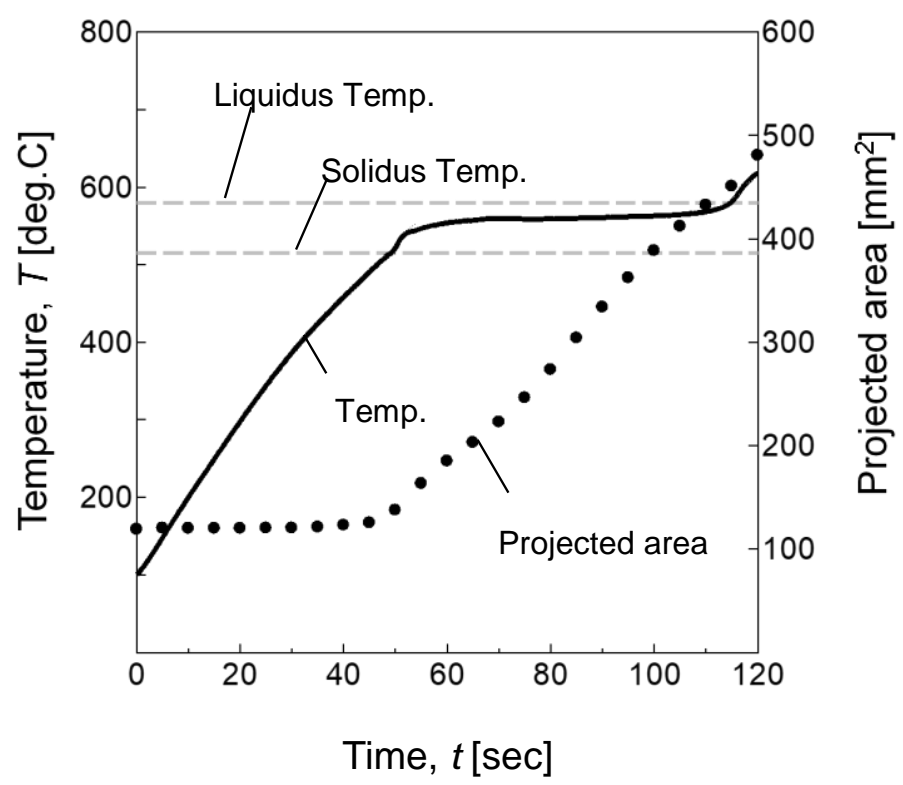

Figure 5. Temperature increase rate vs. change of precursor shape.

The figure clearly demonstrates that precursor formation started almost simultaneously with the phase change, as initially indicated in Figure 4(b), i.e., at approximately $50 \mathrm{~s}$. Normally, the temperature of the blowing process during the thermal decomposition is lower than the melting point of ADC12. Therefore, the blowing agent already gained sufficient energy to blow before the matrix material melted. On the contrary, the stiff Al solid restricted the blowing process, thereby preventing the expansion of the blowing agent particles. Hence, only after the stiff ADC12 solid's melting did the blowing agent expanded successfully, as shown in Figure 4(c) and 4(d). More specifically, the stiff Al solid began melting after it received energy from the light gathered by the elliptical mirror on top of the surface of the precursor. Note that at this time, the internal precursor presented a temperature gradient. Therefore, the precursor 
started to foam only at the upper side of the precursor whereas the lower side of the precursor maintained its original shape, as can be confirmed in Figure 4(c). Generally speaking, the coefficient of $\mathrm{Al}$ thermal conductivity is high; thus, the temperature gradient for such a small piece of the precursor can be ignored. Nonetheless, the higher the input energy to the precursor, the harder it is to neglect the effects of the temperature gradient.

\section{Numerical Results}

The input heat value was calculated from the numerical focus area and power of the halogen lamp. Here, the voltage and current were set at $54 \mathrm{~V}$ and $9.0 \mathrm{~A}$, respectively, whose product equates to the electrical power of $486 \mathrm{~W}$. This power was then divided by the focus area of light with $18-\mathrm{mm}$ of diameter, to provide an input heat value of $3.7 \times 10^{5}$ $\mathrm{W} / \mathrm{m}^{2} 3.7 \times 10^{5} \mathrm{~W} / \mathrm{m}^{2}$. The absorption coefficient was 4.5 , as determined from the preliminary experiment. On one hand, the other values, such as specific heat, density, the volume of precursor, temperature of surrounding wall, and atmospheric temperature, were held constant $[20,21]$. Conversely, the initial values of temperature and melting point of $\mathrm{Al}$ were substituted by the value obtained from the experiment. All these values were substituted into Eq. (5), to obtain the temperature of the precursor. These numerical results were then compared with the experimental results as depicted in Figure 6.

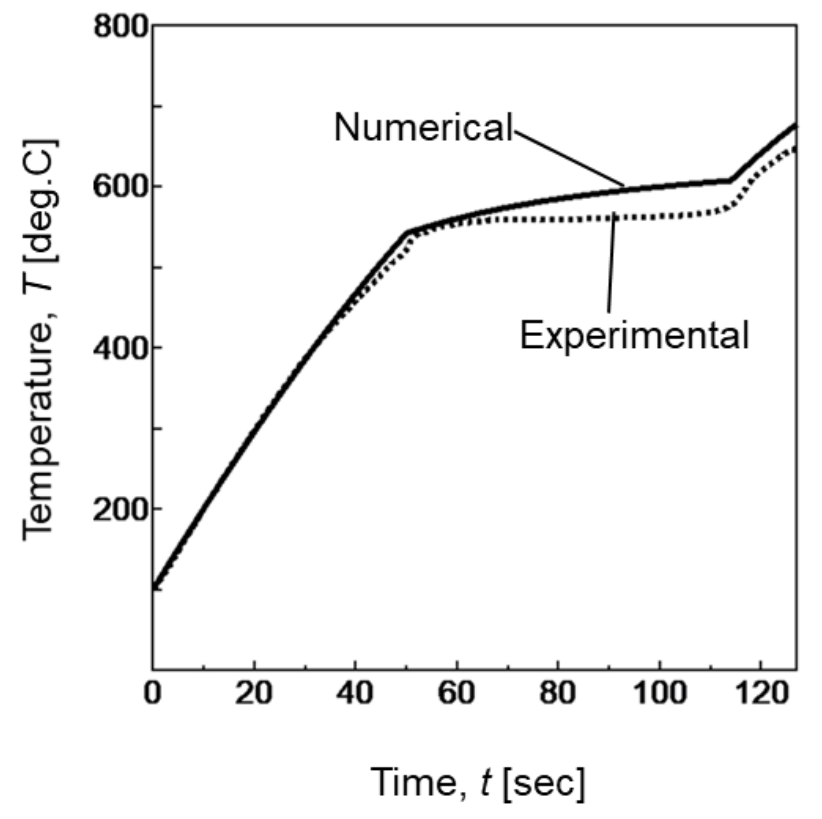

Figure 6. Comparison of experimental and numerical results for precursor.

As can be seen, the numerical results were nearly in agreement with the experimental results until the melting point of $\mathrm{Al}$ at approximately $50 \mathrm{~s}$. Nonetheless, the numerical results also demonstrated slight disagreement with the experimental results after the melting point of $\mathrm{Al}$ has been crossed. Moreover, based on the numerical results, the melting point of $\mathrm{Al}$ appeared slightly earlier than in the experimental results, which could be attributed to the fact that deformation of the Al foam and energy consumption of the blowing agent were neglected, as stated in the preceding texts. In actuality, the energy consumption of the blowing agent should be considered in order to express the changing phenomenon of the particles of the blowing agent into the cavity. In addition, 
the thermal conductivity of $\mathrm{Al}$ foam depends on the porosity and cavity size. Wang et al. [9] found that the effect of thermal conductivity decreases with increasing porosity and cavity size. Therefore, assuming greater thermal conductivity by the gas release of the blowing agent and by the growth of the cavity by the heat treatment is difficult. With respect to the numerical results, the precursor temperature after the melting point of $\mathrm{Al}$ may slightly decrease if the energy consumption of the blowing agent is taken into consideration. This way, an agreement between the numerical and experimental results could be attained.

Figure 7 shows the relation between the numerical temperature and the dependence of each term against the input heat by the halogen lamp in the uniform heating model. That is, this figure expresses the proportion of the lighter side in Eq. (6).

$$
\begin{aligned}
\varepsilon A Q=\rho c V(d T / d t)+A \sigma \varepsilon & \left(T^{4}-T_{0}{ }^{4}\right)+4 A^{\prime} \sigma \varepsilon\left(T^{4}-T_{0}{ }^{4}\right) \\
& +A \alpha_{m, h}\left(T_{w^{-}}-T_{\infty}\right)+4 A^{\prime} \alpha_{m, v}\left(T_{w}-T_{\infty}\right)+L_{a} m\left(d f_{s} / d T\right)(d T / d t)
\end{aligned}
$$

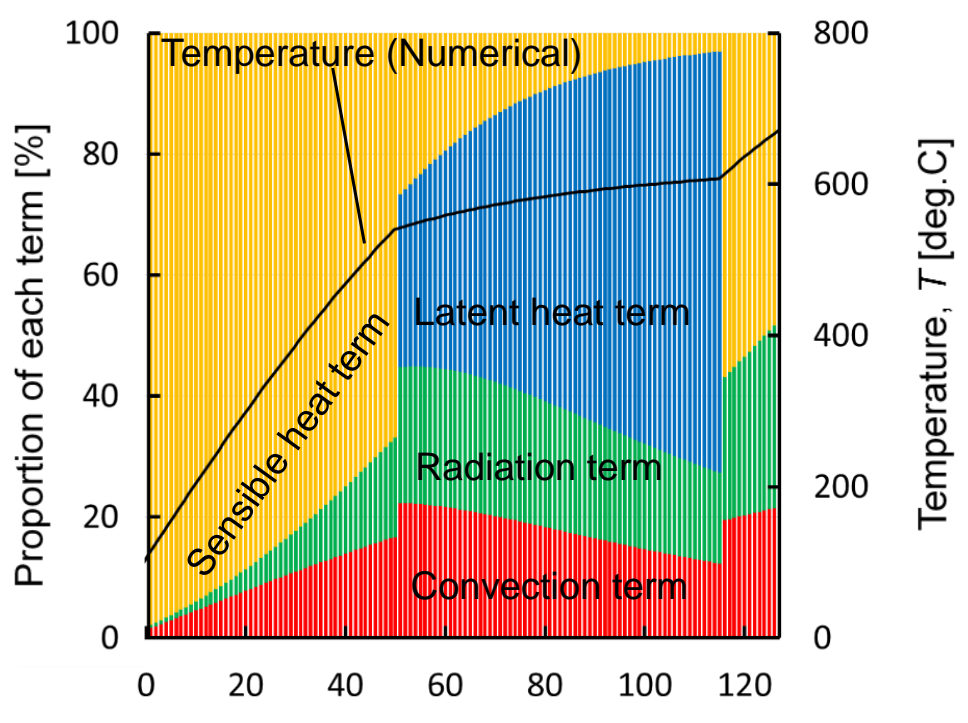

Time, $t[\mathrm{sec}]$

Figure 7. The relation between numerical temperature and dependence of each term on the uniform heating model.

From this figure, the sensible heat term was found to mainly occupy the proportion of Eq. (6) in the beginning. However, the dependence of the sensible heat term was found to decrease by the other terms, i.e., convection, radiation, and latent heat, with increasing time. This can be attributed to the fact that the radiation term and the convection term depend on the temperature difference between the precursor temperature and the atmospheric temperature. Especially in this study, the radiation effect was lager because the precursor was only heated by the light of a halogen lamp. Moreover, the term of latent heat appeared only after the temperature reached the melting point of Al. Nonetheless, it gave larger effects to the change of temperature, as compared to the other terms. On such basis, the precursor temperature could not be raised by the effect of the latent heat term. In actuality, the real phenomenon of latent heat was asymptotic, but a step-change was seen in the figure since the numerical results used the difference method. 
Overall, it was discovered herein that the formation of $\mathrm{Al}$ foam cannot be expressed perfectly using the proposed uniform heating model. Rather, in the future, the effects of deformation and energy consumption of the blowing agent should be considered in the proposed uniform heating model. Nevertheless, this study successfully provided an explanation of the qualitative phenomenon of $\mathrm{Al}$ foam formation. It is also worth noting to consider the thermal conductivity of $\mathrm{Al}$ in identifying the thermal gradient inside the Al foam, given a high input heat value.

\section{CONCLUSION}

The present work introduced a new heat treatment process using light heat sourced from a spot-type halogen lamp to clarify the thermal phenomenon of $\mathrm{Al}$ foam, mainly through experimental and numerical investigations using the uniform heating model. Based on the experimental results, the formation of the precursor most likely initiated at almost the same time as the phase change. A comparison of the experiment and numerical results revealed that the higher the input energy to the precursor, the harder it is to neglect the effects of temperature gradient because the internal precursor would present the temperature gradient.

Accordingly, the numerical results almost corresponded with the experimental results up to the melting point of the Al. After the melting point, the numerical values slightly disagreed with the experimental results. More specifically, the melting point of $\mathrm{Al}$ in the numerical results appeared at a slightly earlier time than in the experiments, which could be explained by the fact that the deformation of Al foam and the energy consumption of the blowing agent were neglected in the uniform heating method. Moreover, with a longer time, the dependence of the sensible heat term was decreased by the other terms, mainly due to the dependence of the radiation term and the convection term on the temperature difference. The latent heat term appeared only after the temperature reached the melting point of $\mathrm{Al}$; nonetheless, it gave larger effects on the change of temperature than the other terms. Conclusively, although the formation of $\mathrm{Al}$ foam cannot be expressed perfectly with the proposed uniform heating model, the model successfully explained a qualitative phenomenon of the formation.

\section{ACKNOWLEDGEMENT}

This research was partially supported by the Matching Planner Program of Japan Science and Technology Agency, JST. The authors would also like to acknowledge Universiti Malaysia Pahang for supporting the research under the postgraduate student mobility program (research attachment mode with Grant No. RDU 1803129).

\section{REFERENCES}

[1] Banhart J. Manufacture, characterisation and application of cellular metals and metal foams. Progress in Materials Science 2001; 46: 559-632.

[2] García-Moreno F. Commercial applications of metal foams: Their properties and production. Materials 2016; 9: 85.

[3] Wang N, Maire E, Cheng Y, et al. Comparison of aluminium foams prepared by different methods using X-ray tomography. Materials Characterization 2018; 138 : 296-307.

[4] San Marchi C, Mortensen A. Deformation of open-cell aluminum foam. Acta 
Materialia 2001; 49: 3959-3969.

[5] Hangai Y, Saito K, Utsunomiya T, et al. Fabrication and compression properties of functionally graded foam with uniform pore structures consisting of dissimilar A1050 and A6061 aluminum alloys. Materials Science and Engineering A 2014; 613: $163-170$.

[6] Lu W, Zhao CY, Tassou SA. Thermal analysis on metal-foam filled heat exchangers. Part I: Metal-foam filled pipes. International Journal of Heat and Mass Transfer 2006; 49: 2751-2761.

[7] Zhao CY, Lu W, Tassou SA. Thermal analysis on metal-foam filled heat exchangers. Part II: Tube heat exchangers. International Journal of Heat and Mass Transfer 2006; 49: 2762-2770.

[8] Zhu X, Ai S, Lu X, et al. Thermal conductivity of closed-cell aluminum foam based on the 3D geometrical reconstruction. International Journal of Heat and Mass Transfer 2014; 72: 242-249.

[9] Wang H, Zhou X, Long B, et al. Thermal properties of closed-cell aluminum foams prepared by melt foaming technology. Transactions of Nonferrous Metals Society of China (English Edition) 2016; 26: 3147-3153.

[10] Banhart J. Light-metal foams - History of innovation and technological challenges. Advanced Engineering Materials 2013; 15: 82-111.

[11] Mukherjee M, Garcia-Moreno F, Banhart J. Defect generation during solidification of aluminium foams. Scripta Materialia 2010; 63: 235-238.

[12] Madhu HC, Kailas S V. Fabrication of localised aluminium foam by a novel polymeric blowing agent. Materials Characterization 2018; 142: 340-351.

[13] Hangai Y, Amagai K, Omachi K, et al. Forming of aluminum foam using steel mesh as die during foaming of precursor by optical heating. Optics and Laser Technology 2018; 108: 496-501.

[14] Hangai Y, Takahashi K, Nagahiro R, et al. Shaping of aluminum foam using point group mold. Materials Transactions 2018; 59: 1952-1955.

[15] Hangai Y, Utsunomiya T, Hasegawa M. Effect of tool rotating rate on foaming properties of porous aluminum fabricated by using friction stir processing. Journal of Materials Processing Technology 2010; 210: 288-292.

[16] Babcsán N, Leitlmeier D, Degischer HP. Foamability of particle reinforced aluminum melt. Materialwissenschaft und Werkstofftechnik 2003; 34: 22-29.

[17] Sato Y, Park S, Matsunaga A, et al. Novel production for highly formable Mg alloy plate. Journal of Materials Science 2005; 40: 637-642.

[18] Sadanari M, Akira M. Engineering of heat transfer. Nisshin Publishing Co., Ltd, 1994.

[19] Shoji I, Koyama S, Inoue M, et al. Mechanical materials science. Maruzen Publishing Co., Ltd, 2014.

[20] Horn V, Kent R. Aluminum Vol.I. Properties, Physical Metallurgy and Phase Diagrams. 1967.

[21] Kumar N, Soni S, Rana RS. Mechanical properties enhancement of Al-Si (ADC12) alloy by heat treatment. International Journal of Mechanical and Production Engineering 2017; 5: 47-51. 